\title{
De la réception d'une città di fondazione
}

Sabaudia 1933-2009

About the Reception of a New Town. Sabaudia 1933-2009

\section{Constance Ringon}

\section{CpenEdition}

Journals

Édition électronique

URL : http://journals.openedition.org/crau/410

DOI : $10.4000 /$ crau. 410

ISSN : 2547-5746

Éditeur

Éditions du patrimoine

\section{Édition imprimée}

Date de publication : 1 décembre 2014

Pagination : 163-172

ISBN : 978-2-7577-0379-3

ISSN : 1296-4077

Référence électronique

Constance Ringon, « De la réception d'une città di fondazione », Les Cahiers de la recherche architecturale et urbaine [En ligne], 30/31 | 2014, mis en ligne le 14 septembre 2017, consulté le 30 avril 2019. URL: http://journals.openedition.org/crau/410; DOI : 10.4000/crau.410 


\title{
De la réception d'une città di fondazione
}

\author{
Sabaudia 1933-2009
}

"Les édifices, les architectures et leurs idées ne sont pas des absolus isolés dans un système clos, mais ils participent d'une activité diachronique de communication. Celle-ci fonde aussi les édifices, l'activité des architectes et les idées qui se développent sur la scène architecturale. L'architecture n'existe et ne dure qu'avec la complicité active de ses publics successifs... Les œuvres ont donc un double rapport qui les lie avec celles qui les précèdent et avec celles qui les suivent et leur donnent du sens ${ }^{1}$. »

Panayotis Tournikiotis

\section{Sabaudia}

Sabaudia, ville "rationaliste », " moderne », " méditerranéenne », qualifiée parfois même de "bolchevique », naît entre 1933 et 1934 sur les rives du lago di Paola, non loin de la mer Tyrrhénienne, à un peu moins d'une centaine de kilomètres au sud de Rome, dans les marais Pontins. Le régime fasciste a entrepris d'assainir cette région marécageuse, en y creusant de nouveaux canaux et en mettant en

1. Panayotis Tournikiotis, extrait du texte de la présentation du hapitre 1 de La Réception de l'architecture du Mouvement moderne: image, usage, héritage, p. 93, $7^{\text {e }}$ conférence internationale de Docomomo, Paris, 16-19 septembre 2002, éditée par Jean-Yves Andrieux et Fabienne Chevallier, Saint-Étienne, Publications de l'université de Saint-Étienne, 2005. 


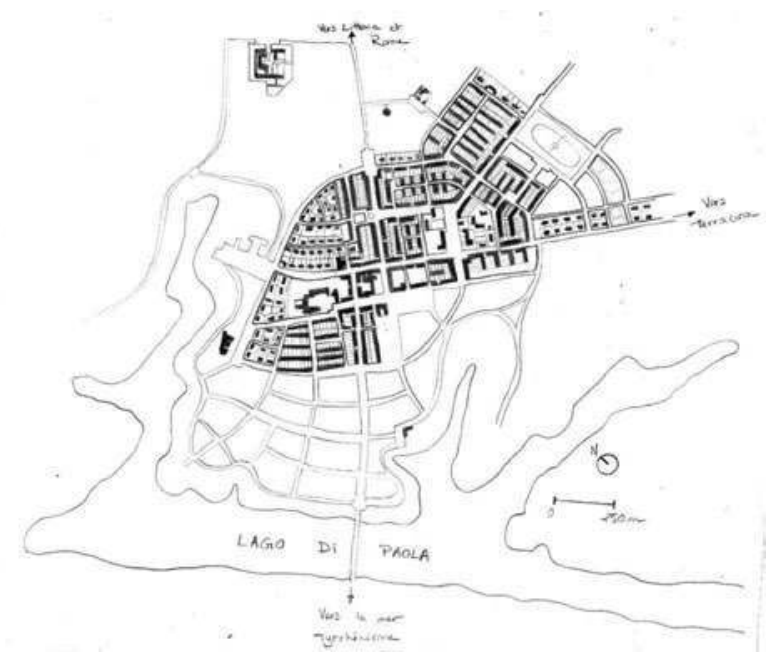

Dessin du plan de Sabaudia d'après le projet de Gino Cancellotti, Eugenio Montuori, Luigi Piccinato et Alfredo Scalpelli (1953).

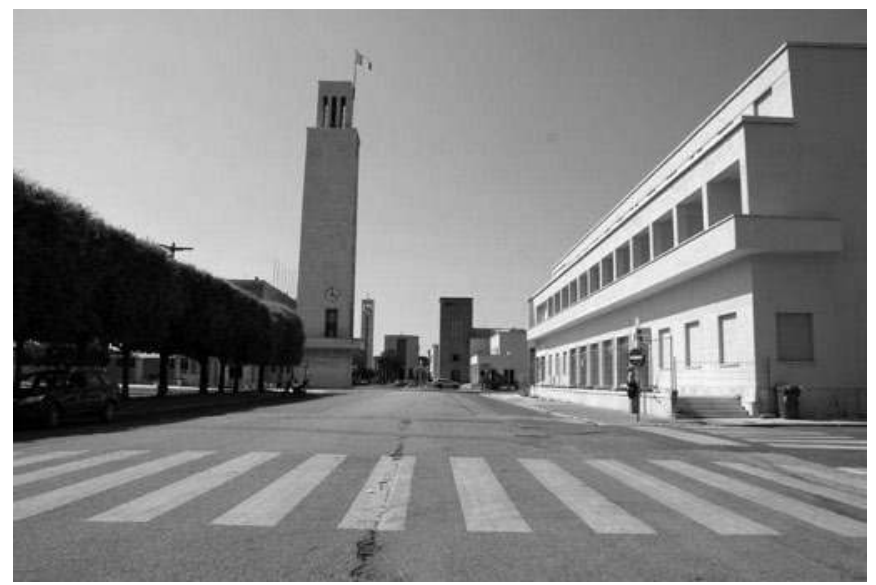

Perspective sur les bâtiments principaux de la ville, du premier au dernier plan : l'auberge, le palazzo del comune, la casa del fascio et l'église.

Photo:

Constance Ringon

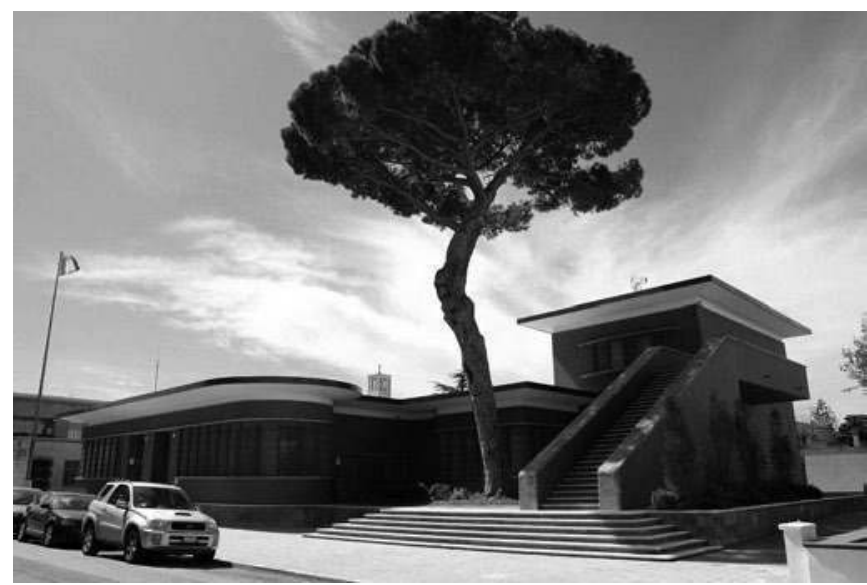


place tout un ensemble d'installations hydrauliques; il y implante des populations issues de régions agricoles surpeuplées du nord de l'Italie auxquelles sont attribuées des fermes et des terres. La décision de créer des villes qui subviendront aux besoins de ces nouveaux habitants n'est prise que dans un deuxième temps. Sabaudia est la deuxième ville à apparaître dans ce territoire inhospitalier, après Littoria ${ }^{2}$.

La ville est bâtie selon un plan « cardo-décumanique » et s'insère dans le projet territorial général composé par l'Opera Nazionale per i Combattenti (ONC), organisation d'anciens combattants (fig. 1). Le site choisi par Valentino Orsolini Cencelli, président de I'ONC et homme politique proche de Mussolini, est sublime: une légère colline qui se déploie au-dessus d'un lac. La ville sera séparée de la mer par celui-ci et par une dune qui file jusqu'au mont Circeo dont le nom vient de la légendaire sorcière qui y aurait résidé.

Une fois le concours lancé et gagné par Luigi Piccinato, Alfredo Scalpelli, Eugenio Montuori et Gino Cancellotti, jeunes architectes fraîchement diplômés, la première pierre est posée le 5 août 1933 par Mussolini lui-même. Deux cent cinquante-deux jours plus tard, le dictateur italien inaugure le centre-ville et annonce la construction d'autres cités, au pas de course. On accède à la nouvelle cité pontine par une via Migliara, qui se dirige vers la mer perpendiculairement à l'antique via Appia. Dans le centre, cet axe se décale en baïonnette, pour laisser place à la majestueuse agora de la ville autour de laquelle se développent différents bâtiments, et repart ensuite en direction du lac, traversant le parc de la ville. Le cœur de la ville est composé des différentes institu- tions nécessaires à la vie d'une commune: palazzo del Comune, casa del Fascio, église, auberge... (Fig. 2) Les logements sont disposés autour du centre, en plusieurs zones. Les bâtiments constitués par des volumes simples et épurés et présentant des ouvertures répétitives correspondent à l'idée courante du visage moderne des constructions des années 1930. Certains édifices repères sont mis en valeur par des volumes particuliers tels la tour du palazzo del Comune ou encore le campanile de l'église. Les volumes dialoguent dans un jeu entre lignes verticales et horizontales, et l'homme ne se sent nullement écrasé par une architecture opprimante qui aurait pu être la métaphore d'un régime totalitaire. Le jaune et le «bleu Savoie " illuminent certaines façades; on entrevoit le mont Circé et la forêt environnante entre les parois de brique et de travertin. On s'y sent, au crépuscule, comme dans un tableau de De Chirico dans lequel les tons foncés auraient été remplacés par de vives couleurs... Quant à l'ambiance, elle est semblable, méditerranéenne, pleine de langueur, mystérieuse et engourdie par le soleil qui cogne sur le sol dont les courbes de niveau ont été vaillamment respectées par les architectes. (Fig. 3)

Mais ce qui est encore plus étonnant avec cette ville, c'est son retentissement toujours d'actualité. L'intérêt qui lui est porté depuis sa construction est passé par différentes phases mais connaît un regain d'enthousiasme depuis le début des années 1970. Comment une ville dont le succès a donné lieu à débats lors de sa création, puis qui s'est vue stigmatisée de par ses origines "fascistes ", peut-elle, maintenant, être perçue comme un chef-d'œuvre? Peut-on dissocier un bâtiment, une ville, du contexte si particulier, de son origine? 


\section{Regards sur Sabaudia}

Dès sa réalisation, les regards qui se portent sur Sabaudia sont marqués par une dualité: le régime fasciste en fait une réalisation emblématique de son action politique et territoriale. Mais c'est aussi une réalisation considérée comme significative par des architectes italiens et étrangers, même si leurs points de vue peuvent être divergents.

Par la suite, l'image de la ville subit le contrecoup de la chute de Mussolini. Jusqu'aux années 1960-1970, elle est très peu évoquée. Alors qu'un architecte originaire de Sabaudia, qui nous emmène visiter sa ville, avoue qu'il n'était guère flatteur de faire état de ce lieu d'origine dans les années 1960, Pier Paolo Pasolini, dans un petit film, vante Sabaudia comme une ville faite par le peuple et pour le peuple italien: «Voici la structure, la forme, le profil d'une ville immergée dans une espèce de lumière lagunaire bien qu'il y ait autour une garrigue splendide. Combien nous avons ri, nous autres, intellectuels, de l'architecture du régime, sur les villes comme Sabaudia. Pourtant maintenant ces villes nous les trouvons absolument inattendues. On sent qu'elles sont faites, comme il est dit un peu rhétoriquement, à mesure d'homme. ${ }^{3}$ »

Le cinéaste Bernardo Bertolucci se rappelle, quant à lui, de la manière dont son regard sur cette ville a changé au cours du temps: "En 1958, j'avais dix-sept ans, Moravia appela mon père et lui dit: pourquoi ne m'accompagnes-tu pas, je vais chercher une maison le long de la mer de Sabaudia, sur les dunes [...] je me souviens comment Alberto et mon père (et moi aussi, les imitant), fûmes offensés par la laideur de l'archi- tecture fasciste de Sabaudia. Flash en avant, vingt ans après, en 1978. Je tourne La Luna, sur cette même plage, et miraculeusement, Sabaudia qui était l'horrible architecture fasciste, est devenue magnifique. Ce sont les mystères de l'évolution du goût, en 1958, l'esthétique ne suffisait pas à sauver un lieu, il y avait le souvenir historique. Aujourd'hui, ce souvenir est délayé, et peut-être avec plus d'objectivité, on tâche d'analyser tout ce qui a été effectivement réalisé et cela vaut la peine de souligner que Sabaudia est devenue l'une des villes de fondation les plus connues internationalement grâce à I'heureux mariage de son plan urbanistique et de son milieu naturel, tout à fait exceptionnel ${ }^{4}$. »

Bruno Zevi, précurseur des nombreux historiens qui vont se pencher sur Sabaudia à partir des années 1980 la cite dans son histoire de l'architecture dès les années $1950^{5}$. Dans les années 2000, la ville commence à mettre en valeur ses édifices. Une patrimonialisation s'ébauche mais elle reste néanmoins fragile malgré la mise en place d'un parcours rationaliste et la restauration de la poste, chef-d'œuvre futuriste de l'architecte Angiolo Mazzoni (fig. 4).

Ces appréciations, dans leurs différences, sont concernées par le long travail de mémoire que doit entreprendre I'Italie sur ce moment de son passé. À partir de l'ensemble d'indices que constituent ces points de vue s'élabore l'idée d'une recherche sur la réception, conduisant à identifier les opinions qui vont concerner Sabaudia et leur variation à travers le temps.

La problématique qui permet de formuler un travail de recherche s'esquisse: Comment évoluent de 1933 jusqu'à aujourd'hui les regards portés sur Sabaudia,
3. Pier Paolo Pasolini et Paolo Brunatto, Pasolini e... la forma della città, Rai TV, 7 février 1974.
4. Dans Monica De Bei Schifano, Gianni Mercurio, Luca Ronchi, Un cielo pieno di cultura. Moravia, Pasolini, Schifano e Sabaudia, Rome, Legenda Aurea, 2003, p. 47.

5. Bruno Zevi, Storia dell'architettura moderna, Turin, Einaudi, 1950, p. 277. 
ville sujette à controverses par l'origine de sa création? Comment ces regards se différencient, s'entrecroisent, se confrontent ou s'ignorent? Quelles en sont les logiques?

\section{Des théories de la réception...}

Depuis longtemps déjà, la notion de réception a été utilisée pour rendre compte du fait que la valeur d'une œuvre naît du rapport qui s'instaure entre elle et ceux qui la considèrent. "Ce sont les regardeurs qui font les tableaux » déclarait Marcel Duchamp ${ }^{6}$. Et Michel Foucault notait au moment de la réédition de L'Histoire de la folie à l'âge classique en 1972 que l'importance de cet ouvrage tenait à la manière dont il avait été reçu: "Quant à la nouveauté, ne feignons pas de la découvrir comme une réserve secrète, comme une richesse d'abord inaperçue: elle n'a été faite que des choses qui ont été dites sur lui et des événements dans lesquels il a été pris ${ }^{7}$. »

Les architectes qui ont développé des recherches en s'appuyant sur cette notion de réception se sont référés au philosophe allemand Hans Robert Jauss qui a élaboré une théorie de la réception en littérature ${ }^{8}$. II ne se limite pas à recenser les jugements énoncés sur

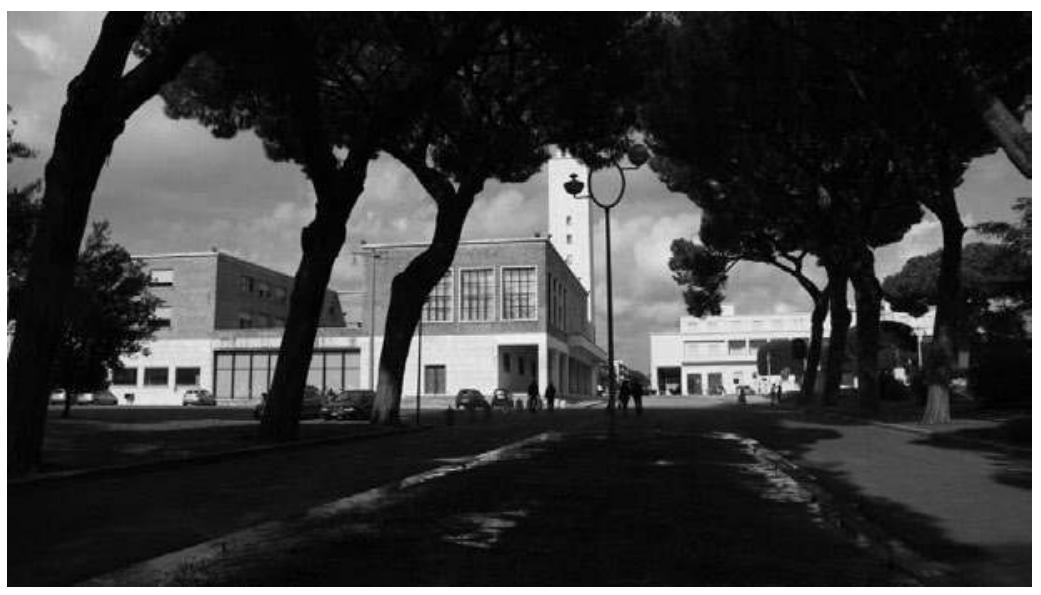

Le palazzo del comune depuis le parc de Saubadia. Photo : Constance Ringon

6. Marcel Duchamp, Marchand du sel, Paris,

Le Terrain vague, 1959.

7. Propos de Michel Foucault rapportés dans un article de Philippe Artières et Mathieu Potte-Bonneville, " Michel Foucault n'est pas un trésor », paru dans Le Monde des livres du 18 mai 2012.

8. Hans Robert Jauss, Pour une esthétique de la réception, traduit de l'allemand, Paris, Gallimard, coll. « Tel », 2001. 
tel ou tel écrit au moment de sa parution. Avec la notion de réception, il construit une théorie de la littérature qui doit permettre d'en écrire une histoire remettant en cause celles qui ont été écrites antérieurement, souvent limitées, selon lui, à l'énumération d'une succession d'œuvres et d'auteurs. En effet pour Jauss, envisager la littérature sous cet angle doit permettre d'identifier les écarts esthétiques autour desquels se constituent en quelque sorte les dynamiques de l'histoire de la littérature. Deux notions, I'horizon d'attente et l'écart esthétique, permettent de comprendre comment la réception de certaines œuvres littéraires peut être marquée par des ruptures.

Plusieurs historiens et théoriciens de l'art et de l'architecture ont également investi, depuis quelques décennies, cette notion de réception dans leurs recherches. On peut citer en particulier les recherches de Michael Baxandall qui a éclairé notre compréhension des peintures de la Renaissance en explicitant les cadres mentaux selon lesquels elles étaient examinées?.

Dans l'étude des pratiques culturelles, des sociologues et des historiens I'ont aussi utilisée. Ils entendaient ainsi rendre compte de la diversité et des clivages qui s'y lisent. Pierre Bourdieu a particulièrement étudié les formes de distinction qui se manifestent dans les choix culturels ${ }^{10}$.

Dans sa volonté de contribuer à la mise en place d'une " histoire culturelle », Pascal Ory donne une place à la notion de réception en la confrontant à celle de fortune critique ${ }^{11}$. La première concernerait plutôt les points de vue qui peuvent être exprimés par les publics dans leur diversité tandis que la seconde désignerait les points de vue développés par des critiques spécialisés ${ }^{12}$. Pierre Vaisse, en revanche propose d'assimiler les deux notions, attribuant leur différence à leur origine, « italienne pour la fortune critique (fortuna critica), germanique pour la réception (rezeption) ${ }^{13}$.

Dans le sillage de la théorie de Jauss, Richard Klein et Philippe Louguet ont réalisé un ouvrage où ils présentent un ensemble de réflexions et d'études dont I'objectif est ainsi défini: "L'évolution des rapports entre l'œuvre et le public, entre l'effet de l'œuvre et sa réception implique l'étude de l'effet produit par l'œuvre elle-même, et de la réception, déterminée par les destinataires ${ }^{14}$. » Ils considèrent que cette démarche peut intervenir directement dans la manière d'élaborer l'histoire de l'architecture: «À l'inverse de l'histoire positiviste et formaliste de l'architecture, la question de la réception inclut le temps et l'usage dans toutes leurs dimensions, implique la distance critique par rapport aux opérateurs et une lecture socialisée de l'architecture. " De son côté, Gérard Monnier souligne comment l'étude de la réception associe « les œuvres, les représentations - l'image - et les actions qui s'en nourrissent ${ }^{16} "$.

Tout en souhaitant que l'analyse en termes de réception s'élargisse aux différentes formes d'art, Vaisse insiste sur des aspects assez particuliers qu'elle présente pour l'architecture: «[...] II existe aussi, en dehors des écrits de critiques ou d'historiens de l'art, une réception de l'architecture, des édifices, des monuments. Elle peut se manifester dans certains cas par la fréquentation. Elle se manifeste aussi par le soin qu'on en prend, par la conservation, la restauration, ou au contraire par la destruction volontaire ${ }^{17}$.
9. Michael Baxandall, L'œil du Quattrocento, Paris, Gallimard, 1985.

10. Pierre Bourdieu, La distinction. Critique sociale du jugement, Paris, Les Éditions de Minuit, 1979.

11. Pascal Ory, L'histoire culturelle, Paris, PUF, coll. «Que-sais-je?», 2004.
12..Ibid. p. 88

13. Pierre Vaisse, "Du rôle de la réception dans I'histoire de l'art », Histoires de l'art,

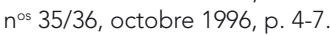

14. Richard Klein et Philippe Louguet (dir.), "La réception en architecture " Cahiers thématiques $n^{\circ} 2$, Lille/Paris, École d'architecture de Lille, Jean-Michel Place, 2002
15. Cahiers thématiques $n^{\circ} 2$, p. 7 .

16. Gérard Monnier, «La réception de I'architecture du Mouvement moderne » dans J.-Y. Andrieu et F. Chevallier (dir.), 2005, op. cit. note 1 .

17. P. Vaisse, «Du rôle de la réception dans I'histoire de l'art », op. cit. note 13. 
Le transfert opéré du champ de la littérature vers l'architecture est intéressant, mais il est nécessaire de noter qu'à partir de celui-ci, le sens donné à la réception se modifie et s'élargit notamment avec Vaisse qui ouvre son étude à la prise en compte de l'ensemble des points de vue et des actions qui s'expriment à l'égard d'une œuvre.

Gérard Monnier rejoint la position de Vaisse en reprenant la définition de Dario Gamboni: la réception, comme objet d'étude général, prend en compte « tout ce qui peut être exprimé à propos d'une œuvre: l'ensemble des relations et des interactions dont les œuvres ont été partie prenante ${ }^{18} »$. En nous appuyant sur l'étude de plusieurs exemples concrets de formes de réception avec les caractéristiques et les différences qui leur sont propres, nous avons construit une grille qui constitue un outil de travail pour l'analyse de la réception de Sabaudia ${ }^{19}$ :

- les acteurs porteurs de ces regards et l'univers de référence qui leur est propre. Ces univers de référence peuvent être d'une grande diversité allant des expériences communes à des références savantes;

- les rencontres et les éventuelles confrontations entre diverses formes de réception d'une même œuvre ;

- l'échelle géographique et spatiale à laquelle se situe le processus de réception étudié ;

- la situation dans le temps des modes de réception permettant de les resituer par rapport à I'histoire de l'édifice concerné;

- les modes d'action et d'intervention sur les édifices qui sont consécutifs à la réception qui en est faite. En effet, celle-ci peut aussi induire des manières $d$ 'intervenir sur l'édifice, mais aussi devenir référence dans les pratiques constructives des architectes.

\section{Premiers temps de la réception}

Situer notre étude de la réception de Sabaudia dans l'optique d'une définition élargie vise à identifier l'ensemble des formes de réception qui sont exprimées sur la ville, dans leurs particularités, leurs différences et leurs confrontations, ceci de la construction de la ville à la commémoration de ses soixante-quinze ans, en 2009.

L'analyse de la diversité des formes de réception devrait permettre d'approfondir la connaissance du sens de l'œuvre, en la situant à plusieurs échelles de I'histoire, celle de l'architecture, mais aussi celle d'une société. La mise en évidence des variations des regards qui se sont portés sur l'architecture de ce lieu peut-elle conduire à en réécrire l'histoire? Notons pourtant que Fabienne Chevallier considère que l'objectif d'une étude en termes de réception, n'est pas de conduire à une réécriture de l'histoire ${ }^{20}$.

La mise en place du travail de recherche a consisté à rassembler l'ensemble des documents ayant trait à Sabaudia dans l'objectif de constituer un corpus d'étude. Cette liste d'ouvrages et de textes, ont a été retenues et ordonnées en une frise de manière chronologique et en fonction des critères choisis: le type d'acteur qui réagit sur la ville, la nature des propos tenus (le contexte de création, la forme architecturale et urbaine, les émotions relatives au paysage, l'évolution voire la sauvegarde du site).

\section{Du regard des architectes aux critiques des politiques}

Le premier temps sélectionné correspond à celui que I'on pourrait qualifier de réception immédiate. Dès que
18. Gérard Monnier, «L'architecture et sa réception », Cahiers thématiques, op. cit. note 14, p. 43. L'article de Dario Gamboni, « Histoire de l'art et « réception »: remarques sur l'état d'une problématique » auquel se réfère Monnier a été publié dans Histoire de l'art, n³5/36, octobre 1996, p. 9-14.
19. Parmi ces études, quelques exemples: Margotte Lamouroux, "Lectures et interprétations de la maison de Wittgenstein $»$, http://specialez.fr/ blog/2010/06/30/lectures-et-intrepretationsde-la-maison-de-wittgenstein/, site consulté le 10 mai 2012;

Caroline Maniaque, «Réception écrite et réception construite: le cas des maisons Jaoul dans la critique corbuséenne anglo-saxonne ", Cahiers thématiques $n^{\circ} 2$, "La réception en architecture », op. cit. note 13; Gilles Ragot (dir.), L'invention d'une ville. Royan années 50, Paris, Éditions du patrimoine, 2003; Adrian von Buttlar, "À Berlin, un château contre un palais", Criticat n`5, Paris, mars 2010.

20. F. Chevallier, «La modernité de l'entre-deux-guerres et l'esthétique de la réception ", dans La Réception de I'architecture du Mouvement moderne: image, usage, héritage, op. cit. note 1. 
s'amorce la réalisation de la ville, et dans les temps qui vont suivre, plusieurs acteurs se manifestent (politiques, architectes, urbanistes...) À plusieurs reprises, Mussolini se rend à Sabaudia durant le chantier, accompagné de nombreuses personnalités. La ville est photographiée, filmée. Le chef de l'État met en scène ce projet d'aménagement territorial, entremêlant les références à la terre, à la valeur du travail physique mais aussi à la modernité.

On a peut-être là en germe, dans cette manière de présenter et de mettre en scène l'architecture, une sorte d'anticipation de ce qui se généralisera vers la fin $\mathrm{du} \mathrm{xx}^{\mathrm{e}}$ siècle, où de nombreuses instances politiques vont jouer dans leurs stratégies des images véhiculées par des édifices très divers. Mais d'autres acteurs se manifestent en prenant des positions qui s'entrecroisent et se confrontent.

Les jeunes architectes qui ont réalisé Sabaudia ont obtenu leurs diplômes dans les années 1920, quand le courant moderne qui se développe dans une grande partie de l'Europe produit des clivages entre les architectes tenant du courant académique et les nouveaux venus dans la profession. Une confrontation a lieu entre les idées défendues par les jeunes architectes en rupture avec les anciens et notamment avec Marcello Piacentini et Gustavo Giovannoni qui occupent des positions importantes dans l'enseignement. Une nouvelle génération revendique un renouveau dans l'architecture à travers la presse et à l'occasion des deux premières expositions rationalistes italiennes qui se déroulent en 1928 et en 1931, à Rome. Lors de la seconde exposition, les critiques sont virulentes. La Tavola degli orrori
( $T$, tableau des horreurs), est exposée par Pietro Maria Bardi, journaliste, historien et critique d'art, qui dénonce les constructions de Piacentini à travers un collage où figurent plusieurs bâtiments jugés académiques. Les architectes débattent et s'affrontent sur leurs conceptions de ce que doit être l'architecture d'État.

Les architectes de Sabaudia, même s'ils ne sont pas nécessairement engagés de façon directe dans ces actions, vont se situer dans ce contexte en tant que jeunes architectes, prônant des formes plus pures que celles de l'époque précédente.

La situation devient plus difficile pour ceux que nous pouvons surnommer ici "jeunes rationalistes" (bien que leur appartenance à un tel courant ne puisse être qualifiée si simplement), quand le débat s'élargit aux politiques. La bourgeoisie romaine qui s'exprime au sein du Parlement ne comprend pas les formes nouvelles défendues par les architectes "modernes" et les conteste. Le débat, très vif, se cristallise autour de trois édifices: la gare de Florence, la cité universitaire de Rome et Sabaudia. Les députés craignent de voir surgir des formes semblables au centre de Rome car un concours est lancé pour la réalisation du pPalazzo Littorio sur la via dell'Impero, avenue qui relie le Colisée à la piazza Venezia. La participation massive d'architectes aux idées nouvelles inquiète. Aucun bâtiment n'y sera cependant construit.

Mussolini prend position. II invite les architectes au pPalazzo Venezia et les défend même publiquement par la voie d'une revue ${ }^{21}$. Quelles sont les raisons de ce soutien? Est-ce de la part du Duce un véritable choix ou est-ce à la suite à de la lecture de l'éditorial d'un étudiant

21. "Discorso al Parlamento », L'Italia Letteraria, a. X, n.23 [n 23 ?], 9 juin 1934 (-XII), p. 2-3. 
anonyme paru dans un journal de lycéens? Cet étudiant est en réalité l'un des fils de Mussolini, fervent admirateur des nouvelles formes d'architecture qui se profilent à travers la réalisation des jeunes architectes en ce début de $\mathrm{xx}^{\mathrm{e}}$ siècle ${ }^{22}$... Mais ce courant moderne sort-il durablement renforcé de ce soutien? Cet appui fait réagir des architectes modernes, exultant à la manière de Giuseppe Pagano, directeur de la revue Casabella, qui tout en fustigeant le " coassement des grenouilles » (ceux qui ont attaqué ces nouvelles constructions) se félicite que "l'Homme qui crée l'Histoire de l'Italie » soutienne les jeunes architectes rationalistes ${ }^{23}$.

L'enthousiasme des jeunes architectes coïncide avec la visite de Le Corbusier à Rome en juin de la même année (1934). À cette occasion, il va donner deux conférences. Il va également visiter les marais Pontins et commenter les nouvelles villes de fondations: Littoria, la première édifiée et Sabaudia qui vient d'être inaugurée. Il tient des propos assez acerbes sur Littoria et son côté cité-jardin, mais il est un peu plus mesuré sur Sabaudia

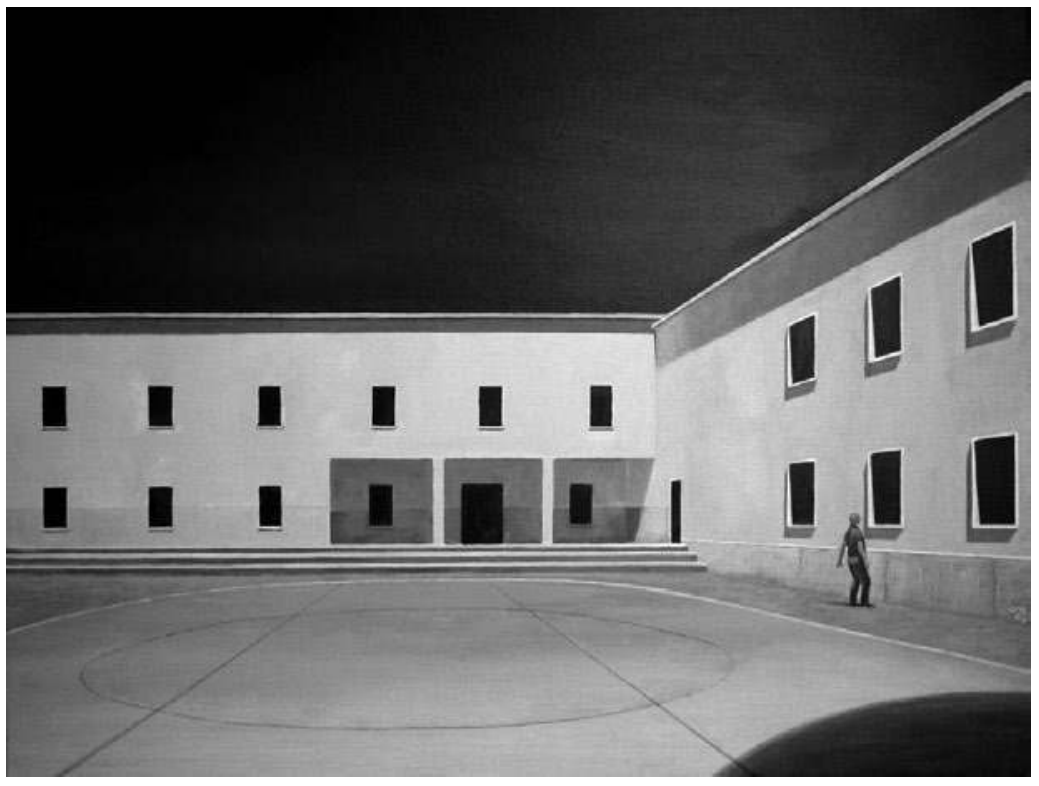

Saubadia, La Sorpresa, de Jean-Michel Léger, 2009.

22. "Fesserie di retrogradi », Anno XII.

Rivista fascista degli studenti, a. VI - n.22 [n²2 ?], 30 mai 1934 (XII1934), FLC U3 (17) 30.

23. Giuseppe Pagano, "Architettura italiana dell'anno XIV », Casabella, 1935. 
bien qu'il la trouve trop « romantique ${ }^{24} »$. Sa visite vise aussi à briguer la commande de la $3^{\text {e }}$ ville construite dans les marais Pontins, Pontinia (fig. 5). Mais sa réalisation sera confiée aux techniciens de I'ONC, notamment en raison des controverses liées à la construction de Sabaudia.

Cesare De Seta rappelait en 1976 que «I'architecture eut un poids sur l'actualité et un caractère de communication tel qu'elle ne pouvait en aucun cas être mise en marge d'une histoire de la culture de la période fasciste $[\ldots]^{25} »$. Sabaudia en offre un exemple dans la manière dont, dès sa réalisation, elle fût propulsée au premier rang de débats dont nous avons énoncé les grandes lignes 26 .

\section{Une recherche en cours...}

Comment les regards vont-ils se recomposer sur la ville pontine et s'en ressaisir, échappant aux enjeux qui avaient marqué le moment de sa création?

Dans I'analyse que Jean-Louis Cohen fait du Mouvement moderne durant le fascisme, il souligne que la dynamique qui s'y est développée ne se clôt pas avec la chute du régime. L'étude des propos tenus sur la cité de fondation à partir des années 1960 devrait prolonger cette réflexion. En effet, des architectes, des intellectuels, des artistes, des historiens s'intéressent à Sabaudia, la resituant dans une culture architecturale globale (fig. 5). Au-delà de la monographie, un portrait de ville se dessine, il s'agit maintenant d'en faire le récit.

24. Le Corbusier, Prélude n 14, 1934.

25. Cesare de Seta, «La culture et I'architecture en Italie entre les deux guerres » dans Silvia Danesi, Luciano Patetta, 1919-1943. Rationalisme et Architecture en Italie, Liège, Pierre Mardaga, 1977 (éd. italienne 1976).

26. Ce qui a été mis en évidence de la réception de Sabaudia ne doit pas faire oublier qu'elle se situe aussi à d'autres échelles, plus modestes. Mario Tieghi retrace dans une série d'entretiens la manière dont les premiers habitants ont occupé cette ville. Mario Tieghi, Sabaudia. Storia viva di una città nei racconti dei protagonisti, Latina, II Gabbiano, 1999.

27. Jean-Louis Cohen, Introduction et adaptation française de Silvia Danesi, Luciano Patetta, 1919-1943, Rationalisme et architecture en Italie, Paris, CERA/Electa France. 